\title{
Cucullanus oceaniensis sp. n. (Nematoda: Cucullanidae) from Pacific eels (Anguilla spp.)
}

\author{
František Moravec $^{1}$ Pierre Sasal $^{2}$, Jürgen Würtz $^{3}$ and Horst Taraschewski ${ }^{3}$ \\ ${ }^{1}$ Institute of Parasitology, Academy of Sciences of the Czech Republic, Branišovská 31, 37005 České Budějovice, Czech \\ Republic; \\ ${ }^{2}$ Centre de Biologie et d'Écologie Tropicale et Méditerranéenne, UMR 5555 CNRS-UP, Parasitologie Fonctionnelle et Evolutive, \\ CBETM, Université de Perpignan, 52 Avenue de Villeneuve, 66860 Perpignan Cedex, France; \\ ${ }^{3}$ Zoologisches Institut der Universität Karlsruhe (TH), Abteilung Ökologie und Parasitologie, Kaiserstrasse 12, 76128 Karlsruhe, \\ Germany
}

Key words: parasitic nematode, Cucullanus, new species, freshwater eel, Anguilla, Wallis and Futuna Islands, Fiji Islands, South Pacific Ocean

\begin{abstract}
A new species of parasitic nematode, Cucullanus oceaniensis sp. n., is described from the intestine of the giant mottled eel Anguilla marmorata (type host) from Futuna Island (Wallis and Futuna Islands, Polynesia) and from A. marmorata and Anguilla sp. (cf. obscura) from Fiji Islands (Melanesia, South Pacific). The main distinguishing characteristics are the length of spicules $(668-1,020 \mu \mathrm{m})$, situation of deirids (slightly anterior to the oesophago-intestinal junction) and the excretory pore (some distance posterior to the end of oesophagus), and the arrangement of caudal papillae in the male. It is the third known species of Cucullanus from Oceania and the first one reported from freshwater eels in the region of South Pacific. Cucullanus faliexae Morand et Rigby, 1998 is considered a junior synonym of Cucullanus australiensis Baylis, 1927.
\end{abstract}

During recent occasional examinations of freshwater eels in the region of Oceania, nematode specimens of a previously unknown species of Cucullanus Müller, 1777 were collected. The new species is described herein.

\section{MATERIALS AND METHODS}

Three specimens ( 2 males and 1 gravid female) of Cucullanus were collected from a giant mottled eel Anguilla marmorata Quoy et Gaimard (body length $73 \mathrm{~cm}$ ) caught in the Vainifao River, Futuna Island (Wallis and Futuna Islands) on 12 October 2004. Two conspecific nematode specimens (1 male and 1 nongravid female) were collected from Anguilla sp. (cf. obscura Günther) from the Fiji Islands in 1995 and eight specimens ( 1 male and 7 females) from $A$. marmorata from the Fiji Islands in July 1996; unfortunately, the only information available for these materials is the host and date of collection (the nematodes were sent from the Fiji Islands to Germany). All nematodes were fixed in $70 \%$ ethanol. For light microscopical examination, the nematodes were cleared with glycerine. Drawings were made with the aid of a Zeiss microscope drawing attachment. After examination, the specimens were briefly placed in $4 \%$ formaldehyde solution and then transferred to $70 \%$ ethanol, in which they were stored. For scanning electron microscopy (SEM), two specimens (male and female from $A$. marmorata from Fiji) from $4 \%$ formalin were postfixed in $1 \%$ osmium tetroxide, dehydrated through a graded ethanol series, critical point dried, and sputter-coated with gold. They were examined with a JEOL JSM-6300 scanning electron microscope at an accelerating voltage of $15 \mathrm{kV}$. All measurements are in micrometres unless otherwise stated.
For comparative purposes, type specimens of Cucullanus australiensis Baylis, 1927 (2 male syntypes, Natural History Museum, London, 1927.8.10.95-98) and C. faliexae Morand et Rigby, 1998 (4 paratypes: 2 males and 2 females, Muséum National d'Histoire Naturelle, Paris, $581 \mathrm{HF}$ ) were examined. The names of fishes follow Froese and Pauly (2005).

\section{DESCRIPTION}

Cucullanus oceaniensis sp. $\mathrm{n}$.

Figs. 1, 2

General: Medium-sized nematodes. Body whitish, slender, with slightly transversely striated cuticle. Lateral alae absent. Oral opening dorsoventrally elongate, surrounded by raised narrow membranous ala (collarette) supported by row of minute basal teeth. Four submedian cephalic papillae and pair of lateral amphids present. Oesophagus muscular, expanded at anterior end to form rather large pseudobuccal capsule (oesophastome); posterior part of oesophagus also expanded, but somewhat narrower than pseudobuccal capsule. Oesophagus opening into intestine through large valve. Nerve ring encircling oesophagus at distance representing $35-49 \%$ of oesophagus length. Deirids small, hooked, slightly asymmetrical, just anterior to oesophago-intestinal junction; excretory pore at short distance below end of oesophagus. Tail conical, with sharply pointed tip.

Male (3 specimens: holotype and 1 paratype from Futuna Island [measurements of latter in parentheses], and 2 paratypes from Fiji [in square brackets]): Length of body 7.14 (9.51) [5.86-7.78] mm, maximum width 

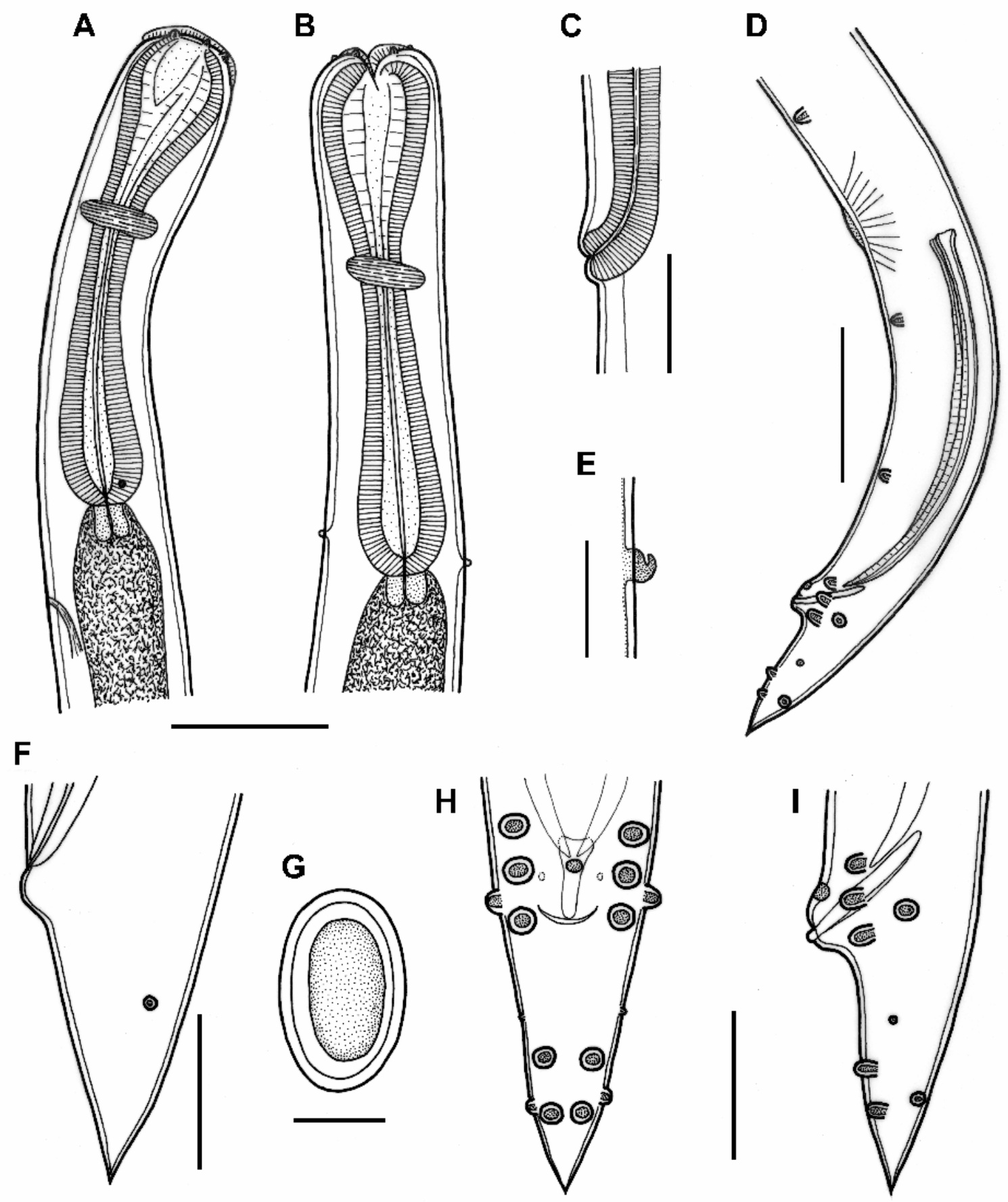

Fig. 1. Cucullanus oceaniensis sp. n. A, B - anterior end of male, lateral and dorsoventral views; C - vulva; $\mathbf{D}$ - posterior end of male, lateral view; $\mathbf{E}$ - deirid; $\mathbf{F}$ - tail of female, lateral view; $\mathbf{G}$ - egg; $\mathbf{H}, \mathbf{I}$ - tail of male, ventral and lateral views. Scale bars: $\mathrm{A}, \mathrm{B}, \mathrm{D}=200 \mu \mathrm{m} ; \mathrm{C}, \mathrm{F}, \mathrm{H}, \mathrm{I}=100 \mu \mathrm{m} ; \mathrm{E}, \mathrm{G}=30 \mu \mathrm{m}$.

190 (326) [299]. Length of entire oesophagus 707 (721) [816-884], length of oesophastome 258 (272) [258299], its width 150 (190) [163-190]; minimum width of oesophagus 68 (95) [68-82]; maximum width of posterior part of oesophagus 122 (177) [136-177]. Oesophagus representing 10 (8) [11-14] \% of whole body length. Distance of nerve ring from anterior extremity 299 (354) [313], representing 42 (49) [35-38] \% of oesophagus length. Deirids and excretory pore 644 (656) [721-739] and 979 (857) [898-1,061], respectively, from anterior end of body. Ventral region of cloacal opening distinctly elevated. Spicules equal, 668 
(990) [819-1,020] long, provided with wide membranous alae. Gubernaculum well sclerotized, 111 (111) [117-135] long, somewhat laterally expanded at proximal end. Ventral preanal sucker well developed. Preanal papillae: 5 subventral pairs, of which first pair well anterior to ventral sucker, second somewhat posterior to ventral sucker, third approximately in mid-way between second pair of papillae and cloacal opening, and fourth and fifth near cloacal aperture; one unpaired, median papilla-like formation present at level of fifth pair of preanals. One pair of lateral adanal papillae present. Postanal papillae: 4 pairs, of which first pair of subventrals just posterior to cloacal opening, second and fourth pairs of subventrals posterior to mid-length of tail and third pair of laterals situated between last two pairs of subventrals. Pair of small lateral papilla-like phasmids present between first and third pairs of postanal papillae. Length of tail 201 (163) [204].

Female (allotype from Futuna Island; measurements of 5 gravid and 1 nongravid specimens [all paratypes] from Fiji in parentheses and square brackets, respectively): Length of body 13.34 (9.49-13.78) [4.65] mm, maximum width 340 (354-503) [190]. Length of entire oesophagus $1,074(1,020-1,088)$ [625], length of oesophastome 313 (313-381) [190], its width 204 (204272) [136]; minimum width of oesophagus 95 (109136) [60], maximum width of its posterior part 177 (190-231) [109]. Oesophagus representing 8 (8-11) [13] \% of whole body length. Distance of nerve ring 394 (408-503) [258] from anterior extremity, representing 37 (39-46) [41] \% of oesophagus length. Deirids and excretory pore $979(870-1,061)$ [516] and 1,278 $(1,170-1,197)$ [734], respectively, from anterior end of body. Length of tail 299 (272-299) [204]; pair of small lateral papilla-like phasmids present near mid-length of tail. Vulva postequatorial, $8.23(5.20-7.66)$ [2.82] $\mathrm{mm}$ from anterior extremity, at 62 (55-59) [61] \% of body length; vulvar lips elevated. Short muscular vagina directed anteriorly from vulva. Uteri opposed. Eggs numerous (eggs absent in 1 paratype); mature eggs oval, thin-walled, with uncleaved contents, 75-81 (75-84) long and 42-45 (42-45) wide; egg wall 6 (6) thick.

T y p e ho s t: Giant mottled eel Anguilla marmorata Quoy et Gaimard, 1824 (Anguillidae, Anguilliformes).

Ot he r host: Anguilla sp. (cf. obscura).

$\mathrm{S}$ ite of infection: Intestine.

T y p e 1 o c a 1 i t y : Vainifao River (14 $30^{\circ} 74^{\prime \prime}$, $\left.178^{\circ} 14 ' 28^{\prime \prime} \mathrm{W}\right)$, Futuna Island (Wallis and Futuna Islands) (A. marmorata).

$\mathrm{O} \mathrm{t} \mathrm{h}$ e $\mathrm{r} \quad 1$ o c a 1 i t y: Fiji Islands (A. marmorata, Anguilla sp. [cf. obscura]).

Prevalence and intensity: A. marmorata, Futuna Island: 3 specimens in one fish examined. A. marmorata, Fiji Islands: 8 specimens in 1 fish. Anguilla sp., Fiji Islands: 2 specimens in unknown number (1 ?) of fish examined.
E t y m o lo g y: The specific name oceaniensis relates to the geographical region of the distribution, i.e., Oceania.

Deposition of type specimens: Holotype, allotype and 3 paratypes in the Helminthological Collection of the Institute of Parasitology in České Budějovice (Cat. No. N-844); one paratype (male) in the Muséum National d'Histoire Naturelle, Paris (Cat. No. 279HG).

\section{DISCUSSION}

The genus Cucullanus Müller, 1777 (Cucullanidae, Seuratoidea) contains a large number of species parasitizing various freshwater, brackish-water or marine fishes around the world; more rarely they are found in aquatic turtles (Petter 1974, Ivashkin and Khromova 1976). Their morphology is rather uniform and some of them have been inadequately described, so that a detailed comparison among all of them is practically impossible. Therefore, some authors prefer to deal with these parasites according to their host groups (Petter 1974) or their zoogeographical region (Moravec et al. 1997, Caspeta-Mandujano et al. 2000, Daniel et al. 2002).

In the description of Cucullanus faliexae Morand et Rigby, 1998 from the marine anguilliform fish Gymnothorax javanicus (Bleeker) from French Polynesia, Morand and Rigby (1998) noted that in addition to $C$. faliexae, there are only four other species of Cucullanus with a protruding cloacal region (considered by them to be a valid taxonomic trait): C. micropapillatus Törnquist, 1931, C. sciaenae Gupta et Gupta, 1979, C. theraponi Rasheed, 1968, and C. laurotravassosi Petter et Le Bel, 1992. However, this feature is characteristic of an additional nine species, of which $C$. australiensis Baylis, 1927, C. filiformis Yamaguti, 1935, C. hians (Dujardin, 1845), C. muraenesocis Yamaguti, 1961, C. murenophidis Campana-Rouget, 1957 and C. robustus Yamaguti, 1935 were described from marine anguilliform fishes.

The morphology and measurements of $C$. faliexae are almost identical with those of $C$. australiensis (only the gubernaculum is reported to be somewhat longer [0.21 $\mathrm{mm}]$ and the excretory pore was not observed in $C$. australiensis) (Baylis 1927, Morand and Rigby 1998). A re-examination of two male syntypes of $C$. australiensis showed the gubernaculum to be 183 and 195 $\mu \mathrm{m}$ long and the excretory pore to be situated somewhat anterior to the end of oesophagus $(1.06 \mathrm{~mm}$ from the anterior extremity). On the other hand, the re-examination of the two male paratypes of $C$. faliexae showed the length of the gubernaculum to be evidently longer (168 and $192 \mu \mathrm{m}$ ) than reported by Morand and Rigby (1998); the length of the gubernaculum given by the latter authors $(110-120 \mu \mathrm{m})$ is probably a mistake, because they illustrated (fig. 1) the gubernaculum about $238 \mu \mathrm{m}$ long, as can be derived from the accompanying scale bar in their paper. The arrangement of male caudal 

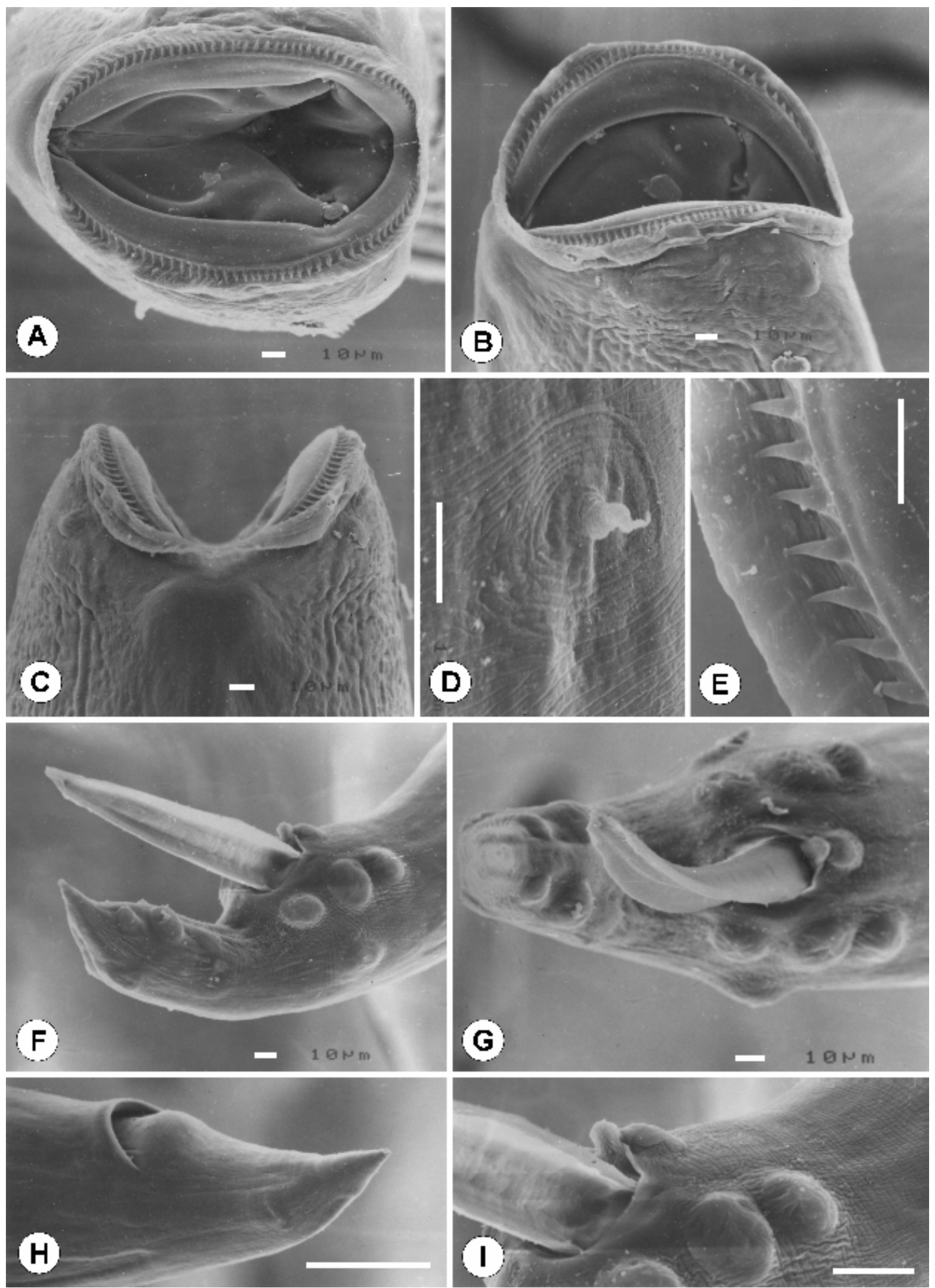

Fig. 2. Cucullanus oceaniensis sp. n., scanning electron micrographs. A, B, C - cephalic end, apical, sublateral and dorsoventral views; D - deirid; $\mathbf{E}$ - detail of cephalic denticles; $\mathbf{F}, \mathbf{G}$ - tail of male, lateral and ventral views; $\mathbf{H}$ - tail of female, lateral view; I - region of cloaca (enlarged), lateral view. Scale bars: D, E $=10 \mu \mathrm{m} ; \mathrm{H}=100 \mu \mathrm{m} ; \mathrm{I}=20 \mu \mathrm{m}$. 
papillae and the situation of deirids were found identical in both species. Since the morphology and measurements of $C$. australiensis and C. faliexae are practically identical (while describing $C$. faliexae, Morand and Rigby [1998] did not compare it with $C$. australiensis) and both species were described from the congeneric fish hosts (Gymnothorax spp.) of the South Pacific (Australia and French Polynesia) with similar areas of distribution (Froese and Pauly 2005), C. faliexae is considered a junior synonym of $C$. australiensis.

Cucullanus oceaniensis differs from C. australiensis in the position of deirids (near the end of the oesophagus vs. just posterior to the nerve ring), shorter spicules (669-1,020 $\mu \mathrm{m}$ vs. $990-1,200 \mu \mathrm{m})$ and the smaller body length (males 5.9-9.5 mm vs. 13-19 mm; gravid females $9.5-13.3 \mathrm{~mm}$ vs. $14-22 \mathrm{~mm}$ ); from $C$. filiformis in the arrangement of caudal papillae and shape of the oesophagus (pseudobuccal capsule distinctly broader than the posterior part of oesophagus vs. pseudobuccal capsule equally broad as the posterior part of oesophagus); and from C. muraenesocis in the arrangement of postanal papillae and situation of the first pair of preanal papillae in relation to the ventral sucker (distinctly anterior to the sucker vs. at the middle of sucker) (Baylis 1927, Yamaguti 1935, 1941, 1961, Morand and Rigby 1998). Moreover, C. oceaniensis has been reported from Polynesia and Melanesia while both $C$. filiformis and $C$. muraenesocis have only been reported from Japan.

In contrast to the new species, $C$. hians has the excretory pore at the level of the oesophagus end, its spicules and the gubernaculum are distinctly longer $(1.26 \mathrm{~mm}$ and $26 \mu \mathrm{m}$, respectively) and it occurs in the Atlantic Ocean and the Mediterranean Sea; C. murenophidis has a different distribution of the preanal and postanal papillae, shorter spicules $(440 \mu \mathrm{m})$ and it occurs near the coast of West Africa; and C. robustus has deirids situated more anteriorly (between the end of oesophagus and the nerve ring), its spicules are distinctly longer $(1.12-1.25 \mathrm{~mm})$, and it was originally described from Japan, but later reported also from New Zealand and the Baltic Sea (Yamaguti 1935, Campana-Rouget 1957, Ivashkin and Khromova 1976).

In contrast to $C$. oceaniensis now reported from freshwater eels (Anguillidae), all the above mentioned species were described from marine anguilliform fishes of the families Congridae and Muraenidae. The only exception is $C$. filiformis originally described from Conger myriaster (Brevoort), but later reported by Yamaguti (1941) from the Japanese eel Anguilla japonica Temminck et Schlegel. Morphologically similar to
C. oceaniensis seems to be Cucullanus anguillae Wang et Ling, 1975, an inadequately described species from Anguilla japonica from China (Wang and Ling 1975), but it has a different arrangement of caudal papillae.

Le-Van-Hoa and Pham-Ngoc-Khue (1967) described a new cucullanid species and genus, Campanarougetia campanarougetae, from Anguilla mauritiana $(=A$. marmorata) from Vietnam; although its gross morphology resembles that of Cucullanus spp., it differs considerably in the structure of the mouth and oesophagus (oral aperture triangular, lumen of oesophagus without pronounced cuticularized armature). In addition, Campanarougetia campanarougetae differs from Cucullanus oceaniensis in the position of the excretory pore at the level of the posterior end of oesophagus, different arrangement of postanal papillae, shorter spicules (48 $\mu \mathrm{m}$ ), and in that the region of the cloacal aperture is not protruding.

Besides C. australiensis (syn. C. faliexae) from French Polynesia (Rangiroa, Tuamotu Archipelago) and C. bourdini Petter et Le Bel, 1992 from marine perciform and tetraodontiform fishes from New Caledonia and French Polynesia (Rangiroa) (Petter and Le Bel 1992, Morand and Rigby 1998), C. oceaniensis is the third known representative of Cucullanus in the southern region of Oceania.

Cucullanus oceaniensis co-occurred with Procamallanus pacificus Moravec, Justine, Würtz, Taraschewski et Sasal, 2005 (Nematoda, Camallanidae) in the freshwater eels of the Futuna Island (Anguilla marmorata) and the Fiji Islands (Anguilla sp.). However, it was absent from the eels (Anguilla obscura Günther, $A$. reinhardtii Steindachner) in New Caledonia, in which Procamallanus pacificus was found (Moravec et al. 2005).

Acknowledgements. Thanks are due to the staff of the Laboratory of Electron Microscopy of the Institute of Parasitology, ASCR, in České Budějovice for their technical assistance and to Irena Husáková from the Department of Helminthology of the same Institute for her help with the preparation of illustrations. Eileen Harris from The Natural History Museum in London and Jimmy Cassone from Muséum National d'Histoire Naturelle in Paris kindly provided type specimens of C. australiensis and C. faliexae, respectively, for study. The work in Futuna Island was financially supported by the CNRS, the French Ministry of Overseas Territories and TOTAL Corporate Foundation. This study was partly supported by the research project (Z60220518) of the Institute of Parasitology, Academy of Sciences of the Czech Republic.

\section{REFERENCES}

BAYLIS H.A. 1927: Some new parasitic nematodes from Australia. Ann. Mag. Nat. Hist., Ser. 9, 20: 214-225.

CAMPANA-ROUGET Y. 1957: Parasites de poissons de mer ouest-africains récoltés par J. Cadenat. Nématodes (4ème note). Sur quelques espèces de Cucullanidae. Révision de la sous-famille. Bull. Inst. Fr. Afr. Noire, Sér. A, 19: 417465.

CASPETA-MANDUJANO J.M., MORAVEC F., AGUILARAGUILAR R. 2000: Cucullanus mexicanus sp. n. (Nematoda: Cucullanidae) from the intestine of the freshwater 
catfish Rhamdia guatemalensis (Pimelodidae) in Mexico. Helminthologia 37: 215-217.

DANIEL V.I., TIMI J.T., SARDELLA N.H. 2002: Cucullanus marplatensis sp. nov. (Nematoda, Cucullanidae) parasitizing Odontesthes argentinensis (Valenciennes, 1835) (Pisces, Atherinidae) from Argentinian waters. Acta Parasitol. 47: 41-46.

FROESE R., PAULY D. (Eds.) 2005: FishBase. World Wide Web electronic publication, www.fishbase.org, version $5 / 2005$.

IVASHKIN V.M., KHROMOVA L.A. 1976: [Cucullanata and Gnathostomatata of animals and man and the diseases caused by them.] Osnovy nematodologii 27. Nauka, Moscow, 436 pp. (In Russian.)

LE-VAN-HOA, PHAM-NGOC-KHUE 1967: Relation entrée les Seuratoidea et les Cucullanidae par l'intermédiaire d'un nouveau nématode Campanarougetia campanarougetae n. g., n. sp., parasite des anguilles du Sud-VietNam. Bull. Soc. Pathol. Exot. 60: 393-398.

MORAND S., RIGBY M.C. 1998: Cucullanin nematodes from coral reef fishes of French Polynesia, with a description of Cucullanus faliexae n. sp. (Nematoda: Chitwoodchabaudiidae). J. Parasitol. 84: 1213-1217.

MORAVEC F., JUSTINE J.-L., WÜRTZ J., TARASCHEWSKI H., SASAL P. 2005: Procamallanus (Procamallanus) pacificus n. sp. (Nematoda: Camallanidae) from Pacific eels (Anguilla spp.). J. Parasitol. (In press.)

MORAVEC F., KOHN A., FERNANDES B.M.M. 1997: New observations on seuratoid nematodes parasitic in fishes of the Paraná River, Brazil. Folia Parasitol. 44: 209-223.

PETTER A.-J. 1974: Essai de classification de la famille des Cucullanidae. Bull. Mus. Natl. Hist. Nat., $3^{\mathrm{e}}$ série, No. 255, Zool. 177: 1469-1490.

PETTER A.-J., LE BEL J. 1992: Two new species in the genus Cucullanus (Nematoda - Cucullanidae) from the Australian region. Mem. Inst. Oswaldo Cruz 87 (Suppl. 1): 201-206.

WANG P., LING X. 1975: Some nematodes of the suborder Camallanata from Fujian Province, with notes on their life histories. Acta Zool. Sin. 21: 350-358. (In Chinese, Engl. summary.)

YAMAGUTI S. 1935: Studies on the helminth fauna of Japan. Part 9. Nematodes of fishes, 1. Jpn. J. Zool. 6: 337-386.

YAMAGUTI S. 1941: Studies on the helminth fauna of Japan. Part 33. II. Nematodes of fishes. Jpn. J. Zool. 9: 343-396.

YAMAGUTI S. 1961: Studies on the helminth fauna of Japan. Part. 57. Nematodes of fishes, III. J. Helminthol., R.T. Leiper Suppl., pp. 217-228. 